\title{
The Application of CRISPR/Cas9 Technology in the Management of Genetic and Nongenetic Plant Traits
}

\author{
Wilbert Mutezo $\mathbb{D}^{1},{ }^{1}$ Moosa M. Sedibe, ${ }^{1}$ Alina Mofokeng, ${ }^{2}$ Nemera Shargie, ${ }^{2}$ \\ and Tegwe Soko ${ }^{3}$ \\ ${ }^{1}$ Central University of Technology, Free State Private Bag X 20539, Bloemfontein 9300, South Africa \\ ${ }^{2}$ Agricultural Research Council-Grain Crops, Private Bag X 1251, Potchefstroom 2520, South Africa \\ ${ }^{3}$ Rattary Arnold Research Station, P.O. Box CH 142, Chisipiti, Harare, Zimbabwe \\ Correspondence should be addressed to Wilbert Mutezo; wmutezo@gmail.com
}

Received 1 April 2021; Accepted 24 June 2021; Published 6 July 2021

Academic Editor: Allen Barker

Copyright (C) 2021 Wilbert Mutezo et al. This is an open access article distributed under the Creative Commons Attribution License, which permits unrestricted use, distribution, and reproduction in any medium, provided the original work is properly cited.

\begin{abstract}
Gene editing (GE) has yielded positive results in the management of genetic and nongenetic plant traits. Clustered interspaced palindromic repeats (CRISPR) and CRISPR-associated proteins (Cas) (together known as the CRISPR/Cas system) are relatively easy to use, compared to other existing genome editing technologies. The CRISPR/Cas tool produces unchanging gene mutations, with the ability to segregate from the Cas9/sgRNA construct to avoid similar modifications by CRISPR/Cas. CRISPR/Cas GE is fast and accurate; crops developed using this technique are resistant to viruses, fungi, and bacteria and resilient to abiotic and biotic stresses, while crops established using conventional methods take between 10 and 15 years to develop resistance. Therefore, CRISPR/Cas is a useful tool for sustainable agricultural production. Plant traits have been successfully manipulated using this technology. Notwithstanding the technical challenges of transferring CRISPR/Cas9 developed crops from the laboratory to the field, additional obstacles include uncertain policy systems, dispute over intellectual property ownership, and acceptability by consumers. Several Cas9-based applied techniques have gained popularity that enable researchers to enhance plants with speed and accuracy. In conclusion, the CRISPR/Cas9 system is a powerful technology for genetically modifying crops.
\end{abstract}

\section{Introduction}

Gene engineering enhances the heritable or nonheritable genetic material to control the genotype or phenotype of a certain cell, tissue, or organism. It is used to delete and insert a specific gene or DNA sequence to yield genetic adjustments [1]. Gene editing (GE) has benefits for the management of both genetic and nongenetic plant traits, and clustered interspaced palindromic repeats (CRISPR) and CRISPR-associated proteins (Cas) (the CRISPR/Cas system) have shown considerable advantages due to their simplicity and specificity [2-4].

CRISPR/Cas systems have been well determined and studied in animal species, but much remains to be carried out in plants [5]. The system has arisen to substitute conventional plant breeding and transgenic (GMO) strategies for plant improvement. Zinc finger nucleases (ZFNs) and TAL effector nucleases (TALENs) have traditionally been the only methods available for double-stranded DNA cleavage. However, execution of these methods is complex and hence the need for simpler and effective alternatives [6]. Recently, a less challenging method was developed, based on the bacterial type II CRISPR/Cas immune system, the CRISPR/Cas9 system. The CRISPR/Cas system allows specific DNA cleavage and DNA repair through nonhomologous end joining (NHEJ) and homologous directed repair (HDR) mechanisms [7].

\section{The Evolution of CRISPR/Cas9 Technology}

Considerable research has focused on CRISPR systems. The essential CRISPRs were identified decades ago by Ishino in 
Escherichia coli [8]; however, the functions of the system remained unclear until the mid-2000s.

In 1993, CRISPRs were detected in Haloferax mediterranei $[7,9-11]$ and were successively recognized in a range of bacterial and archaeal genomes. In the early 2000s, the detection of sequence resemblance between the spacer regions of CRISPRs and sequences of bacteriophages, archaeal viruses, and plasmids shed light on CRISPR as an immune system [12]. This important discovery, made by Mojica and others [13], was not recognized, but was published in 2005 with the aid of three research organizations.

Proportional genomic analyses have demonstrated that CRISPR/Cas systems are bacterial cell immune response systems against viral invasion [11]. The first CRISPR was discovered in E. coli, using an iap gene evaluation [3]. The preserved gene sequence was repeated five times, with consistent spacing: subsequent genome analysis revealed a 14-time repeat. Jansen et al. [14] suggested the term CRISPR, and this became established as the research community worked on these sequences [7].

In nature, CRISPR/Cas systems function as a prokaryotic acquired immune system [13]. Insertion of a phage sequence into the spacer region of the Staphylococcus thermophilus CRISPR rendered this strain resistant to the equivalent phage. In-depth examination of genomic islands has revealed that Cas1-solo genes are consistently positioned within the locality of B DNA polymerases [15]; therefore, it is believed that Cas1 is the main enzyme responsible for the movement of important genetic elements, named casposons [16].

\section{CRISPR/Cas9 Technology in Plant GE}

The ability to develop GE crops using CRISPR/Cas is comparable to those established using conventional or mutation breeding. Depending on the situation, there may be other suitable tools; however, in general, CRISPR is recognized as a highly suitable tool.

Evolving gene modification research in crop plants involves probing gene function and rewiring regulatory signaling networks and the single-guide RNA (sgRNA) library for high-throughput loss of function screening [17-19]. The influential and innovative CRISPR/Cas system could be used to enhance plant adaptation to local environmental stresses and maximize crop yields. The Cas9 protein cleavages DNA, producing a double-strand break (DSB) and activating a mobile DNA restoration mechanism. If there is no homologous repair, the error-prone NHEJ pathway is prompted leading to random insertions/deletions or even substitutions at the DSB site, causing mutations on this region [19].

Alternatively, if a homologous template is present, the HDR pathway is instigated, resulting in gene mutations comprising gene knock-in, deletion, or mutation [20]. The use of CRISPR in plants is very topical; three reports defined the first use of Cas9/sgRNA technology in plant gene engineering [21]. These were shortly followed by a further five studies, which added gene mutations via NHEL and HDR, and generated whole plants carrying mutations at the targeted loci $[9,17,22,23]$. Gene mutations added via NHEJ and HDR have been documented. Five of the numerous reports generated whole plants that carry mutations at the targeted loci [17].

The CRISPR/Cas tool produces unchanging gene mutations, with the ability to segregate from the Cas9/sgRNA construct to avoid similar modifications by CRISPR/Cas $[4,15,24-26]$.

CRISPR/Cas technology has been applied to many plant structures to promote resistance to environmental stresses, while enhancing other agronomic characteristics. Although advances of this system have increased on-target effectiveness, most works are in its infancy and, therefore, needs improvement. Nonetheless, CRISPR/Cas9-based genome editing is a promising approach to develop "suitably edited" plants that will assist with future food security.

A different technology has been established that enables DSB and donor template-free base editing from $\mathrm{C}$ to $\mathrm{T}$ at a specific target site [27]. These base substitutions are mediated by a cytidine deaminase that converts cytidine into uridine by eliminating an amino group. Consequent DNA repair of the U-G mismatch results in a U-A base pair that is further resolved to a stable T-A base pair [27]. CRISPR/Casbased directed evolution is a developing field, in which base editing plays a key role, and in recent years, numerous groups have established methods to improve base editing [27-29]. The viability and effectiveness of cytidine deaminase base editing (CBE) have been tested in rice at three chosen targets: one target (P2) in OsPDS, which encodes a phytoene desaturase, and two targets (S3 and S5) in OsSBEIIb, which encode starch branching enzyme IIb. Accurate point mutations were effectively introduced into the three target sites, and the efficiencies of obtaining the desired mutations at the S5, S3, and P2 targets were $19.2 \%, 10.5 \%$, and $1.0 \%$, respectively [30].

The CRISPR/Cas base editing tool enables specific and efficient single-base conversion at targeted genomic sites and has been extensively used in several organisms, including plants [31-33]. Nonetheless, many obstacles limit the prevalent use of base editors, such as high off-target activity, limited PAM sites, and wide editing windows; various attempts have been made in recent years to reduce these limitations and improve the specificity of base editors $[34,35]$. In the future, further research is required for modifying existing CBE and ABE tools, applying the REPAIR and RESCUE systems in plants, and developing novel base editors with the capacity of directing transversion mutations [30].

Base editing is widely used in developing disease-resistant crops. Notwithstanding the previously described limitations above, base editing systems have the potential to simplify crop bioproduction or improvement, through high precision and efficiency [36]. Base editing could play a dynamic role in the introduction of gene mutations and directed protein evolution. Cytosine base editing lacking UGI has the capacity to make diverse mutations, other than C-to-T. This ability has already been used in CRISPR-X and targeted AID-mediated mutagenesis- (TAM-) based studies to identify known and novel mutations in mammalian cells 
in cancer therapeutics targets PSMB5 and BCR-ABL, respectively [36]. Although there is an ever-increasing interest in $\mathrm{BE}$ and $\mathrm{PE}$ editing tools, there remain challenges that require further research, one major challenge being constrained target sites due to the PAM specificity of Cas proteins $[31,33]$.

\section{Websites for Developing Constructs for CRISPR/Cas9 Genetic Engineering Procedures}

For the best outcomes from CRISPR-based trials, some essential components must be considered in the experimental design. The general objective of CRISPR experiments is to acquire high rates of ideal genome mutations, low rates of off-targets or nonspecific impacts, and a suitable readout of the outcome [13]. While CRISPR has demonstrated high success rates, the fluctuations in effectiveness and particularity are not perfect, and delivery of the CRISPR framework into the natural model system of interest is challenging in certain frameworks [13]. Hence, it is important to streamline and validate exploratory designs to achieve the best outcomes.

\section{Methods to Improve the Delivery of CRISPR/ Cas9 in Plants}

Plant transformation approaches vary significantly, and it is difficult to clearly distinguish in planta and in vitro transformation. GE provides an opportunity to create a transgene-free edited plant genome. The delivery method of the CRISPR/Cas9 segment is of critical significance for genome modification in plants [37]. Diverse delivery techniques, for example, Agrobacterium-mediated, bombardment or biolistic methods, floral-dip, and PEG-mediated protoplasts are often applied to crops for proficient genome editing. The efficiency of Agrobacterium-mediated transfer was improved by avoiding chemical selection, such as kanamycin. The absence of a chemical selection agent following Agrobacterium infection gives greater rates of plant regeneration, compared to using chemical selection and, therefore, likely improves mutant callus/shoot production [38].

For bombardment or biolistic methods, in planta GE has been reported, in which callus culture and regeneration are not required; shoot apical meristems (SAMs) were used that contained a subepidermal cell layer, L2, from which germ cells later develop during floral organogenesis [39]. For the biolistic delivery, gold particles coated with plasmids expressing CRISPR/Cas9 components were bombarded into SAM-exposed embryos of imbibed seeds and mutations were achieved [39]. In Arabidopsis, the easiest and most commonly used method of in planta transformation is the floral-dip [40]. This technique is based on the immersion of unopened flowers into an Agrobacterium suspension containing a strong surfactant, generally Silwet-77. Owing to the surfactant, the Agrobacterium cells enter flower tissues and transfer T-DNA into plant cells, predominantly into the gametes. Following fertilization, zygotes with inserted T-DNA give rise to transgenic embryos and, after germination, to transgenic plants. This method makes it very easy to generate transgenic plants, without going through in vitro mechanisms [40].

\section{Mechanisms Underlying CRISPR/Cas9- Mediated Resistance}

6.1. CRISPR/Cas9 and Disease Resistance. The use of resistance genes in conventional breeding has resulted in crops that use hypersensitive response mechanisms to fight disease [41]. There have been numerous advances in the use of functional mutations to create broad-spectrum disease resistance, as in the Arabidopsis thaliana DMR6 gene [41]. Inactivation of the AtDMR6 homologue in tomatoes confers the plant with resistance to Pseudomonas syringae, Xanthomonas spp., and Phytophthora capsici [41].

Wang et al. [42] developed mutagenized rice lines using CRISPR/Cas9 technology that showed significantly better blast resistance compared to wild-type rice [19]. The development of wheat varieties resistant to powdery mildew was achieved through mutation of the TaMLO-A1, TaMLO$\mathrm{B} 1$, and TaMLO-D1 genes in the wheat genome using CRISPR/Cas9 [43].

Successful gene mutations of the phytoene desaturase gene in cassava using CRISPR/Cas 9 confirmed its use as a versatile GE tool for this crop. CRISPR/Cas9-mediated modification of the cassava elF4E isoforms, $n$ CBP- 1 and $n$ CBP-2, resulted in increased CBSD resistance [44]. CRISPR/Cas9/sgRNA technology has also been used for altering canker tolerance genes in Duncan grapefruit. This provides evidence that CRISPR/CAs technology provides a useful technique to induce resistance in plants [45].

6.2. CRISPR/Cas9 and Virus Resistance. Improved virus resistance was achieved in Cucumis sativus L. by disrupting the recessive eukaryotic translation initiation thing $4 \mathrm{E}$ (eIF4E) gene [46]. Nontransgenic heterozygous eif4e mutant plants were chosen for the production of nontransgenic homozygous T3 plants. Conversely, nonmutant and/or heterozygous plants showed susceptibility to viruses.

6.3. CRISPR/Cas9 and Parasitic Weed Resistance. Gene silencing using RNA interference (RNAi) has been successful in preventing haustorial initiation and growth in tobacco [47] and lettuce [48], respectively. In maize, RNAi did not result in Striga resistance [49], whereas viral-induced gene silencing in maize for Striga hermonthica was successful [50]. Mutant alleles at the low germination stimulant 1 (LGS1) locus significantly decrease Striga germination activity [51]. Advances in the production of reference genomes for parasitic species require greater use of GE technologies to interfere with genes responsible for parasitism [52].

\section{Latest Achievements in Practical Uses of CRISPR/Cas9 Technology in Plants}

The useful deletion of a candidate gene is a simple stage in plant genetics. The Cas9/gRNA technique has been used 
efficiently for performing gene knockouts in plants, such as Arabidopsis, rice, tobacco, and sorghum [51, 53-56]. CRISPR/Cas9-mediated genome editing has been demonstrated commercially in different plants, such as maize [57-59], wheat [60-64], soybean [56], tomato [65-68], petunia [23], and sorghum [51]. Twenty-four plant species have been transformed using CRISPR/Cas technology: cereal crops (barley, maize, rice, switchgrass, and wheat), vegetables and melons (cabbage, carrot, cassava, cucumber, potato, rape, tomato, and watermelon), fruits (apple, banana, grape, grapefruit, and orange), legumes (alfalfa and soybean), technical plants (Ethiopian mustard, cotton, and flax), and coffee [18].

Using CRISPR/Cas9 technology, deletions of $200 \mathrm{bp}$ have been executed with a frequency of $>10 \%$ in rice protoplasts [28], and deletions of 357-761 bp have been conducted at frequencies of $4-45 \%$ in rice protoplasts and up to $6 \%$ in T0 flowers [67].

The type II CRISPR/Cas system from Streptococcus pyogenes and its simplified derivative, the Cas9/sgRNA system, have emerged as powerful new tools for targeted gene knockout in bacteria, yeast, fruit fly, zebrafish, and human cells. Diversification of these structures has led to expression of the Cas9/sgRNA system in two dicot plant species, Arabidopsis and tobacco, and two monocot crop species, rice and sorghum. Agrobacterium tumefaciens was used for delivery of genes encoding Cas9, sgRNA, and a nonfunctional mutant inexperienced fluorescence protein (GFP) to Arabidopsis and tobacco [53]. The mutant GFP gene contained target sites in its 5' coding regions that were effectively cleaved by a CAS9/sgRNA complex, along with error-prone DNA repair, resulting in functional GFP genes. DNA sequencing validated Cas9/sgRNA-mediated mutagenesis at the target site.

\section{Advantages of CRISPR/Cas9 Technology in Plant Genetic Engineering}

Studies of different plant systems have demonstrated the utility, versatility, and heritability of CRISPR/Cas9-mediated GE. Numerous reports have also shown that necessary crop characteristics can be altered by single-gene mutations using CRISPR/Cas9 [69]. The system is easy to develop, design, and implement, since only one nuclease is required and the gRNA is easy to assemble and transfer into cells [70]. Numerous reports have shown no off-target mutations, making CRISPR/Cas9 the tool of preference for plant genome engineering $[23,67,71]$. Compared with the time consuming and high-cost cloning procedures of ZFNs and TALENs, the CRISPR/Cas system allows for low cost and rapid adaptation to a number of sites and produces mutations of high frequencies for plant genomes [28, 37, 72, 73]. Several studies have confirmed the potential of CRISPR/Cas to generate a large range of allelic diversity at unique loci $[42,58,73]$.

\section{Limitations to Using CRISPR/Cas9 in Plants}

Undertaking CRISPR/Cas9-mediated GE requires a number of materials upfront, for example, laboratory resources, proper identification of genomic targets, appropriate vectors that precisely and effectively cut target sites, and a robust regeneration protocol and transformation system [69]. The major setback of CRISPR/Cas9, if not properly designed and executed, is elevated off-targets $[54,74]$. Some of the limitations for utilizing CRISPR in plants are as follows:

(i) Direct targeting of $S$ genes may cause a fitness cost due to their linkage with other desirable genes, particularly genes controlling plant growth and development

(ii) "Off-target mutations" have become a mainstay in efforts to improve the CRISPR system, particularly in the production of transgene-free crops

(iii) Safety and commercialization issue are directly related to humans and other living organisms

\section{Off-Target Consequences in CRISPR/Cas- Mediated GE}

In terms of the frequency of off-target edits that can arise from crop genome editing, it is imperative to recognize the comparative source and scale of genome alterations that occur in plants under natural circumstances. Irrespective of the source, mutations in higher plants can result in errorprone repairs, some of which may prompt direct toxic effects to reduce protein synthesis, destroy cell membranes and photosynthetic proteins to inhibit plant growth, lead to chromosome fusions, or produce genetic changes in plant populations that may be passed on to the next generations $[75,76]$.

The DSBs in coding regions presented by CRISPR/Cas9, or other genome-editing reagents, are also contingent on plant endogenous repair pathways that may insert or delete fragments to prompt mutation. Consequently, genome alterations by CRISPR/Cas9, or other genome-editing reagents, are much more site-specific than related modifications by traditional plant breeding methods, and those DSBs in off-target sites are equivalent to naturally induced mutations $[37,74,77]$. In relation to process-based risks, further improvements in targeting, off-target molecular characterization, and the implementation of methodologies to avoid or characterize foreign DNA in plant germplasms in crop breeding programs will reduce the undesired outcomes of the genome-editing process. Additional characterization of downstream risks connected with a specific genome-edited crop should be conducted, as appropriate for the generated phenotype generated and its anticipated uses [78].

\section{Policy and Legislation Hindering the Expansion of GE Technology}

GE represents a multidimensional shift beyond "genetic modification" (GM) and is outpacing both public understanding and the capacity of regulatory organizations. Under EU law, most products produced using mutagenesis are considered GMOs by definition, but are explicitly exempted from GMO regulation. A debate exists regarding whether 
mutagenesis exemption, which dates from 2001, only applies to processes available until 2001 or if the exemption also includes future processes [79]. The introduction of genomeedited products that are nearing commercialization in agriculture has underscored that the US biotechnology regulatory system has not kept pace with technological advances. Of the three agencies that regulate engineered crops and animals for agriculture, only one has indicated how it will regulate edited plants [80].

The Food and Drug Administration can regulate any plant, but has not indicated if it will single out edited plants; the US Department of Agriculture currently has no authority over edited plants when the edit is a deletion or does not contain any added DNA from a plant pest [80]; and depending on how the statutes are interpreted, the Environmental Protection Agency might be able to regulate plants edited to tolerate pests and diseases. Labeling requirements also remain undefined. Regardless, industry and some consumer groups are anxious over editing technology and may be the ultimate arbiters of whether edited products make it to market [80]. Canada's science-based regulatory system was adapted for GM crops in the early 1990s and has proven sufficiently robust in responding to new plant breeding techniques, having approved two varieties of GE canola [81].

\section{Regulatory Issues on GM Crops and Biotechnology-Related Products in an African Context}

Recognizing the important (potential) benefits of biotechnology to improving food security and rural development, governments across Africa have taken steps to establish a policy framework to support and enable the adoption of biotechnology, including GM crops and derived products. A more detailed analysis of relevant policies and regulations is presented. Examples of recent policy decisions regarding GM crops include the following: approvals for general release and commercial variety registration for insect-resistant, GM cotton hybrids in Ethiopia (2018), Kenya (2019), Malawi (2019), and Nigeria (2018). While farmers in Ethiopia started planting GM cotton on a limited scale in 2019, GM seed distribution in Kenya, Nigeria, and Malawi will start in 2020 [82, 83].

Apart from reigniting old ethical debates, GE also poses a particular challenge to legal practitioners as this new technology does not always fit into existing definitional molds, and it lacks clear legal precedent. In this article, the most salient areas of concern in the South African legal context are identified [84].

\section{Why Edited Plants Should Not be Considered Transgenic}

Transgene-free techniques can be achieved by CRISPRmediated plant GE, for example, by isolating out transgenes through segregation, delivering the ribonucleoprotein complex of Cas9 and gRNA through particle bombardment or using a protoplast system, and utilizing viral vectors for editing germline cells [85]. This ensures that the end product does not carry any foreign DNA and hence cannot be regarded as transgenic. The recently acquired capacity to precisely alter plant genomes by adjusting native genes without presenting new hereditary material offers new chances to quickly exploit natural variation, create new variation, and make changes, with the objective of creating more productive and nutritious plants [86]. It has been proposed that NHEJ will be a favored methodology in crop breeding that utilizes GE, in light of the fact that the resultant plants are considered to hold no transgenes, which is one of the significant concerns of GM crops. In view of this, transgene-free plants developed through GE must not be regulated as GMO. A few specialists and interest groups have also proclaimed that such transgene-free crops should not be regulated under procedure-based GMO guidelines [87, 88].

\section{CRISPR Regulatory Issues}

Predictable target mutagenesis in plants, with the aid of the CRISPR/Cas9 system, opens up new avenues in plant research. Traditional arbitrary mutagenesis strategies are unable to contact every gene for inactivation due to the randomness of the gene combination.

CRISPR/Cas9 technology can assist in producing changes on unapproachable genes, transforming multiple loci, and producing huge deletions, which result in quick advances in plant breeding efforts, without introducing transgenes [11, 89-91]. While GM crops may help crop improvement, the arguments regarding the environmental and health consequences of GM plants could be prevented through GE technology (Belhaj et al., 2013; Botha \& Viljoen, 2008). CRISPR-edited plants are excluded from current GMO regulations; however, this technology may present new issues, with regards to legislation and consumer acceptance of GE plants [9, 22], Shan et al., 2013.

Crops that have been engineered at the gene/genome level through the use of CRISPR/Cas9 technology are not regarded as GMO under product-based GMO regulations due to the fact that the ultimate products are not GMOs [17]. GE plants with the proper governing arrangements in place may be deemed more appropriate than plants that bring foreign DNA in their genomes. The US Department of Agriculture has specified that GE plants that do not contain foreign DNA will not be viewed as GMOs, while the European Commission is expected to soon submit their view regarding the regulatory ambiguity of genome editing (Belhaj et al., 2013). The potential for CRISPR technology to be utilized in plant research and crop breeding, and its value for global food security, is also contingent on consumer acceptance of GE crops $[1,17]$.

\section{Conclusions and Perspectives}

Recent studies have demonstrated that CRISPR/Cas9 technology is an important system for genome engineering. Several Cas9-based applied techniques are being utilized in plants, and these tools will deliver unique understanding 
into plant biology and enable rapid and precise plants enhancements via breeding.

As genome-editing technology is used more extensively in plants, the safety of genome-edited plants is a point of discussion in the plant community [92]. Undoubtedly, the CRISPR/Cas9 system is one of the most powerful tools in crop genetic modification.

\section{Abbreviations}

CRISPR: Clustered random interspaced palindromic repeats

NHEJ: Nonhomologous end joining

HDR: Homologous directed repair

GE: $\quad$ Gene editing

GM: Genetic modification.

\section{Conflicts of Interest}

The authors declare that they have no conflicts of interest.

\section{Acknowledgments}

The authors would like to acknowledge the effort of all the coauthors of this study, as well as journal edit for assisting in language edits. The authors would also like to thank Seed Co Private Ltd., Zimbabwe, for offering their research facilities, and to the Agricultural Research Council of South Africa for providing germplasm. The authors would like to thank the Central University of Technology for funding these studies, which facilitated this research.

\section{References}

[1] F. Wolter, P. Schindele, and H. Puchta, "Plant breeding at the speed of light: the power of CRISPR/Cas to generate directed genetic diversity at multiple sites," BMC Plant Biology, vol. 19, no. 1, p. 176, 2019.

[2] S. N. Kieper, C. Almendros, and S. J. J. Brouns, "Conserved motifs in the CRISPR leader sequence control spacer acquisition levels in type I-D CRISPR-Cas systems," FEMS Microbiology Letters, vol. 366, no. 11, p. fnz129, 2019.

[3] A. Maikova, V. Kreis, A. Boutserin, K. Severinov, and O. Soutourina, "Using an endogenous CRISPR-Cas system for genome editing in the human pathogen Clostridium difficile," Applied and Environmental Microbiology, vol. 85, no. 20, pp. e01416-e01419, 2019.

[4] M. A. Price, R. Cruz, S. Baxter, F. Escalettes, and S. J. Rosser, "CRISPR-Cas9 in situ engineering of subtilisin E in Bacillus subtilis," PLoS One, vol. 14, no. 1, p. e0210121, 2019.

[5] X. Liu, C. Xie, H. Si, and J. Yang, "CRISPR/Cas9-mediated genome editing in plants," Methods, vol. 121-122, pp. 94-102, 2017.

[6] N. Toro, M. R. Mestre, F. Martínez-Abarca, and A. GonzálezDelgado, "Recruitment of reverse transcriptase-cas1 fusion proteins by type VI-A CRISPR-Cas systems," Frontiers in Microbiology, vol. 10, p. 2160, 2019.

[7] D. K. Sahel, A. Mittal, and D. Chitkara, "CRISPR/Cas system for genome editing: progress and prospects as a therapeutic tool," Journal of Pharmacology and Experimental Therapeutics, vol. 370, no. 3, pp. 725-735, 2019.
[8] S. E. Klompe, P. L. H. Vo, T. S. Halpin-Healy, and S. H. Sternberg, "Transposon-encoded CRISPR-Cas systems direct RNA-guided DNA integration," Nature, vol. 571, no. 7764, pp. 219-225, 2019.

[9] X. Ji, H. Zhang, Y. Zhang, Y. Wang, and C. Gao, "Establishing a CRISPR-Cas-like immune system conferring DNA virus resistance in plants," Nature Plants, vol. 1, p. 15144, 2015.

[10] G. Faure, S. A. Shmakov, W. X. Yan et al., "CRISPR-Cas in mobile genetic elements: counter-defence and beyond," $\mathrm{Na}$ ture Reviews Microbiology, vol. 17, no. 8, pp. 513-525, 2019.

[11] Z. Guo, D. Sun, S. Kang et al., "CRISPR/Cas9-mediated knockout of both the PxABCC2 and PxABCC3 genes confers high-level resistance to Bacillus thuringiensis Cry1Ac toxin in the diamondback moth, Plutella xylostella (L.)," Insect Biochemistry and Molecular Biology, vol. 107, pp. 31-38, 2019.

[12] A. C. de Oliveira Luz, J. M. A. da Silva, A. M. Rezende, M. P. S. de Barros, and T. C. Leal-Balbino, "Analysis of direct repeats and spacers of CRISPR/Cas systems type IF in Brazilian clinical strains of Pseudomonas aeruginosa," Molecular Genetics and Genomics, vol. 294, no. 5, pp. 1095-1105, 2019.

[13] R. A. Wilkinson, C. Martin, A. A. Nemudryi, and B. Wiedenheft, "CRISPR RNA-guided autonomous delivery of Cas9," Nature Structural \& Molecular Biology, vol. 26, no. 1, pp. 14-24, 2019.

[14] R. Jansen, J. D. A. van Embden, W. Gaastra, and L. M. Schouls, "Identification of a novel family of sequence repeats among prokaryotes," OMICS: A Journal of Integrative Biology, vol. 6, no. 1, pp. 23-33, 2002.

[15] K. Xie and Y. Yang, "RNA-guided genome editing in plants using a CRISPR-Cas system," Molecular Plant, vol. 6, pp. 1975-1983, 2013.

[16] K. S. Makarova, Y. I. Wolf, J. Iranzo et al., "Evolutionary classification of CRISPR-Cas systems: a burst of class 2 and derived variants," Nature Reviews Microbiology, vol. 18, no. 2, pp. 67-83, 2020.

[17] K. Belhaj, A. Chaparro-Garcia, S. Kamoun, N. J. Patron, and V. Nekrasov, "Editing plant genomes with CRISPR/Cas9," Current Opinion in Biotechnology, vol. 32, pp. 76-84, 2015.

[18] A. Scheben, Y. Yuan, and D. Edwards, "Advances in genomics for adapting crops to climate change," Current Plant Biology, vol. 6, pp. 2-10, 2016.

[19] L. Wang, L. Wang, Q. Tan et al., "Efficient inactivation of symbiotic nitrogen fixation related genes in Lotus japonicus using CRISPR-Cas9," Frontiers in Plant Science, vol. 7, p. 1333, 2016.

[20] W. L. Yeo, E. Heng, L. L. Tan et al., "Characterization of Cas proteins for CRISPR-Cas editing in streptomycetes," Biotechnology and Bioengineering, vol. 116, no. 9, pp. 2330-2338, 2019.

[21] Q. Shan, Y. Zhang, K. Chen, K. Zhang, and C. Gao, "Creation of fragrant rice by targeted knockout of the OsBADH2 gene using TALEN technology," Plant Biotechnology Journal, vol. 13, no. 6, pp. 791-800, 2015.

[22] S. Khatodia, K. Bhatotia, N. Passricha, S. M. P. Khurana, and N. Tuteja, "The CRISPR/Cas genome-editing tool: application in improvement of crops," Frontiers in Plant Science, vol. 7, p. 506, 2016.

[23] Y. Zhang, Y. Bai, G. Wu et al., "Simultaneous modification of three homoeologs of TaEDR1 by genome editing enhances powdery mildew resistance in wheat," The Plant Journal, vol. 91, no. 4, pp. 714-724, 2017.

[24] J. Basak and C. Nithin, "Targeting non-coding RNAs in plants with the CRISPR-Cas technology is a challenge yet worth accepting," Frontiers in Plant Science, vol. 6, p. 1001, 2015. 
[25] M. Nieves-Cordones, S. Mohamed, K. Tanoi et al., "Production of low-Cs ${ }^{+}$rice plants by inactivation of the $\mathrm{K}^{+}$ transporter Os HAK 1 with the CRISPR-Cas system," The Plant Journal, vol. 92, pp. 43-56, 2017.

[26] W. Wang and X. Wang, "Single-cell CRISPR screening in drug resistance," Cell Biology and Toxicology, vol. 33, no. 3, pp. 207-210, 2017.

[27] N. Capdeville, P. Schindele, and H. Puchta, "Application of CRISPR/Cas-mediated base editing for directed protein evolution in plants," Science China Life Sciences, vol. 63, no. 4, pp. 613-616, 2020.

[28] Y. Lu and J.-K. Zhu, "Precise editing of a target base in the rice genome using a modified CRISPR/Cas9 system," Molecular Plant, vol. 10, no. 3, pp. 523-525, 2017.

[29] N. Soda, L. Verma, and J. Giri, "CRISPR-Cas9 based plant genome editing: significance, opportunities and recent advances," Plant Physiology and Biochemistry, vol. 131, pp. 2-11, 2018.

[30] S. S. Bharat, S. Li, J. Li, L. Yan, and L. Xia, "Base editing in plants: current status and challenges," The Crop Journal, vol. 8 , no. 3, pp. 384-395, 2020.

[31] Y. Li, W. Li, and J. Li, "The CRISPR/Cas9 revolution continues: from base editing to prime editing in plant science," Journal of Genetics and Genomics, vol. 5, pp. 1673-8527, 2021.

[32] R. Yarra and L. Sahoo, "Base editing in rice: current progress, advances, limitations, and future perspectives," Plant Cell Reports, vol. 40, pp. 595-604, 2021.

[33] C. Zhang, Y. Wang, F. Wang et al., "Expanding base editing scope to near-PAMless with engineered CRISPR/Cas9 variants in plants," Molecular Plant, vol. 14, no. 2, pp. 191-194, 2021.

[34] T.-K. Huang and H. Puchta, "Novel CRISPR/Cas applications in plants: from prime editing to chromosome engineering," Transgenic Research, pp. 1-21, 2021.

[35] S. D. Rodrigues, M. Karimi, L. Impens et al., "Efficient CRISPR-mediated base editing in Agrobacterium spp," Proceedings of the National Academy of Sciences of the United States of America, vol. 118, p. e2013338118, 2021.

[36] Abdullah, Z. Jiang, X. Hong, S. Zhang, R. Yao, and Y. Xiao, "CRISPR base editing and prime editing: DSB and templatefree editing systems for bacteria and plants," Synthetic and Systems Biotechnology, vol. 5, pp. 277-292, 2020.

[37] L. Arora and A. Narula, "Gene editing and crop improvement using CRISPR-Cas9 system," Frontiers in Plant Science, vol. 8, p. 1932, 2017.

[38] L. Chen, W. Li, L. Katin-Grazzini et al., "A method for the production and expedient screening of CRISPR/Cas9-mediated non-transgenic mutant plants," Horticulture Research, vol. 5, pp. 1-12, 2018.

[39] H. Hamada, Y. Liu, Y. Nagira, R. Miki, N. Taoka, and R. Imai, "Biolistic-delivery-based transient CRISPR/Cas9 expression enables in planta genome editing in wheat," Scientific Reports, vol. 8, no. 1, p. 14422, 2018.

[40] N. E. Zlobin, M. V. Lebedeva, and V. V. Taranov, "CRISPR/ Cas9 genome editing through in planta transformation," Critical Reviews in Biotechnology, vol. 40, no. 2, pp. 153-168, 2020.

[41] D. P. de Toledo Thomazella, Q. Brail, D. Dahlbeck, and B. Staskawicz, "CRISPR-Cas9 mediated mutagenesis of a DMR6 ortholog in tomato confers broad-spectrum disease resistance," bioRxiv, 2016.

[42] M. Wang, Y. Lu, J. R. Botella, Y. Mao, K. Hua, and J.-k. Zhu, "Gene targeting by homology-directed repair in rice using a geminivirus-based CRISPR/Cas9 system," Molecular Plant, vol. 10, no. 7, pp. 1007-1010, 2017.

[43] S. Wang, S. Zhang, W. Wang, X. Xiong, F. Meng, and X. Cui, "Efficient targeted mutagenesis in potato by the CRISPR/Cas9 system," Plant Cell Reports, vol. 34, no. 9, pp. 1473-1476, 2015.

[44] M. A. Gomez, Z. D. Lin, T. Moll et al., "Simultaneous CRISPR/ Cas9-mediated editing of cassava eIF4E isoforms nCBP-1 and nCBP-2 reduces cassava brown streak disease symptom severity and incidence," Plant Biotechnology Journal, vol. 17, no. 2, pp. 421-434, 2019.

[45] H. Jia, Y. Zhang, V. Orbović et al., "Genome editing of the disease susceptibility gene CsLOB1 in citrus confers resistance to citrus canker," Plant Biotechnology Journal, vol. 15, no. 7, pp. $817-823,2017$.

[46] J. Chandrasekaran, M. Brumin, D. Wolf et al., "Development of broad virus resistance in non-transgenic cucumber using CRISPR/Cas9 technology," Molecular Plant Pathology, vol. 17, no. 7, pp. 1140-1153, 2016.

[47] A. Alakonya, R. Kumar, D. Koenig et al., "Interspecific RNA interference of shoot meristemless-like disrupts Cuscuta pentagona plant parasitism," The Plant Cell, vol. 24, no. 7, pp. 3153-3166, 2012.

[48] A. A. Tomilov, N. B. Tomilova, T. Wroblewski, R. Michelmore, and J. I. Yoder, "Trans-specific gene silencing between host and parasitic plants," The Plant Journal, vol. 56, no. 3, pp. 389-397, 2008.

[49] G. Ejeta and J. Gressel, Integrating New Technologies for Striga Control: Towards Ending the Witch-Hunt, World Scientific, Singapore, 2007.

[50] D. Kirigia, S. Runo, and A. Alakonya, "A virus-induced gene silencing (VIGS) system for functional genomics in the parasitic plant Striga hermonthica," Plant Methods, vol. 10, no. 1, p. 16,2014

[51] D. Gobena, M. Shimels, P. J. Rich et al., "Mutation in sorghum low germination stimulant 1 alters strigolactones and causes striga resistance," Proceedings of the National Academy of Sciences, vol. 114, no. 17, pp. 4471-4476, 2017.

[52] J. H. Westwood, C. W. Depamphilis, M. Das et al., "The parasitic plant genome project: new tools for understanding the biology of Orobanche and Striga," Weed Science, vol. 60, no. 2, pp. 295-306, 2012.

[53] W. Jiang, H. Zhou, H. Bi, M. Fromm, B. Yang, and D. P. Weeks, "Demonstration of CRISPR/Cas9/sgRNA-mediated targeted gene modification in arabidopsis, tobacco, sorghum and rice," Nucleic Acids Research, vol. 41, no. 20, p. e188, 2013.

[54] T. Arazoe, K. Miyoshi, T. Yamato et al., "Tailor-made CRISPR/Cas system for highly efficient targeted gene replacement in the rice blast fungus," Biotechnology and Bioengineering, vol. 112, no. 12, pp. 2543-2549, 2015.

[55] S. E. Anami, Y.-M. Zhang, L.-X. Zhang, L. Zhu, and H.-C. Jing, "Sorghum genetic transformation: current status and future target traits," Biotechnology of Major Cereals, CABI, Wallingford, UK, 2016.

[56] J. Mammadov, R. Buyyarapu, S. K. Guttikonda, K. Parliament, I. Y. Abdurakhmonov, and S. P. Kumpatla, "Wild relatives of maize, rice, cotton, and soybean: treasure troves for tolerance to biotic and abiotic stresses," Frontiers in Plant Science, vol. 9, p. 886, 2018.

[57] S. Svitashev, C. Schwartz, B. Lenderts, J. K. Young, and A. Mark Cigan, "Genome editing in maize directed by CRISPR-Cas9 ribonucleoprotein complexes," Nature Communications, vol. 7, no. 1, p. 13274, 2016.

[58] J. Shi, H. Gao, H. Wang et al., "ARGOS8 variants generated by CRISPR-Cas9 improve maize grain yield under field drought 
stress conditions," Plant Biotechnology Journal, vol. 15, no. 2, pp. 207-216, 2017.

[59] A. T. Shayanowako, M. Laing, H. Shimelis, and L. Mwadzingeni, "Resistance breeding and biocontrol of Striga asiatica (L.) Kuntze in maize: a review," Acta Agriculturae Scandinavica, Section B-Soil \& Plant Science, vol. 68, no. 2, pp. 110-120, 2018.

[60] Y. Wang, X. Cheng, Q. Shan et al., "Simultaneous editing of three homoeoalleles in hexaploid bread wheat confers heritable resistance to powdery mildew," Nature Biotechnology, vol. 32, no. 9, pp. 947-951, 2014.

[61] J. Gil-Humanes, Y. Wang, Z. Liang et al., "High-efficiency gene targeting in hexaploid wheat using DNA replicons and CRISPR/Cas9," The Plant Journal, vol. 89, pp. 1251-1262, 2017.

[62] P. Bhowmik, E. Ellison, B. Polley et al., "Targeted mutagenesis in wheat microspores using CRISPR/Cas9," Scientific Reports, vol. 8 , no. 1, p. $6502,2018$.

[63] D. Kim, B. Alptekin, and H. Budak, "CRISPR/Cas9 genome editing in wheat," Functional \& Integrative Genomics, vol. 18, no. 1, pp. 31-41, 2018.

[64] M. A. Ali, M. Shahzadi, A. Zahoor et al., "Resistance to cereal cyst nematodes in wheat and barley: an emphasis on classical and modern approaches," International Journal of Molecular Sciences, vol. 20, no. 2, p. 432, 2019.

[65] C. Brooks, V. Nekrasov, Z. B. Lippman, and J. Van Eck, "Efficient gene editing in tomato in the first generation using the clustered regularly interspaced short palindromic repeats/ CRISPR-associated9 system," Plant Physiology, vol. 166, no. 3, pp. 1292-1297, 2014.

[66] Y. Ito, A. Nishizawa-Yokoi, M. Endo, M. Mikami, and S. Toki, "CRISPR/Cas9-mediated mutagenesis of the RIN locus that regulates tomato fruit ripening," Biochemical and Biophysical Research Communications, vol. 467, no. 1, pp. 76-82, 2015.

[67] V. Nekrasov, C. Wang, J. Win, C. Lanz, D. Weigel, and S. Kamoun, "Rapid generation of a transgene-free powdery mildew resistant tomato by genome deletion," Scientific Reports, vol. 7, no. 1, p. 482, 2017.

[68] V. K. Bari, J. A. Nassar, S. M. Kheredin et al., "CRISPR/Cas9mediated mutagenesis of carotenoid cleavage dioxygenase 8 in tomato provides resistance against the parasitic weed Phelipanche aegyptiaca," Scientific Reports, vol. 9, no. 1, p. 11438, 2019.

[69] S. M. Schaeffer and P. A. Nakata, "CRISPR/Cas9-mediated genome editing and gene replacement in plants: transitioning from lab to field," Plant Science, vol. 240, pp. 130-142, 2015.

[70] M. M. Mahfouz, A. Piatek, and C. N. Stewart Jr., "Genome engineering via TALENs and CRISPR/Cas9 systems: challenges and perspectives," Plant Biotechnology Journal, vol. 12, no. 8, pp. 1006-1014, 2014.

[71] Y. Mao, H. Zhang, N. Xu, B. Zhang, F. Gou, and J.-K. Zhu, "Application of the CRISPR-Cas system for efficient genome engineering in plants," Molecular Plant, vol. 6, pp. 2008-2011, 2013.

[72] Y. Ding, H. Li, L.-L. Chen, and K. Xie, "Recent advances in genome editing using CRISPR/Cas9," Frontiers in Plant Science, vol. 7, p. 703, 2016.

[73] Y. Demirci, B. Zhang, and T. Unver, "CRISPR/Cas9: an RNAguided highly precise synthetic tool for plant genome editing," Journal of Cellular Physiology, vol. 233, no. 3, pp. 1844-1859, 2018.
[74] M. B. Begemann, B. N. Gray, E. January et al., "Precise insertion and guided editing of higher plant genomes using Cpf1 CRISPR nucleases," Scientific Reports, vol. 7, no. 1, p. 11606, 2017.

[75] Y. Naito, K. Hino, H. Bono, and K. Ui-Tei, "CRISPRdirect: software for designing CRISPR/Cas guide RNA with reduced off-target sites," Bioinformatics, vol. 31, no. 7, pp. 1120-1123, 2015.

[76] R. Singh, C. Kuscu, A. Quinlan, Y. Qi, and M. Adli, "Cas9chromatin binding information enables more accurate CRISPR off-target prediction," Nucleic Acids Research, vol. 43, no. 18, p. e118, 2015.

[77] M. H. U. Khan, S. U. Khan, A. Muhammad, L. Hu, Y. Yang, and C. Fan, "Induced mutation and epigenetics modification in plants for crop improvement by targeting CRISPR/Cas9 technology," Journal of Cellular Physiology, vol. 233, no. 6, pp. 4578-4594, 2018.

[78] H. Zhao and J. D. Wolt, "Risk associated with off-target plant genome editing and methods for its limitation," Emerging Topics in Life Sciences, vol. 1, no. 2, pp. 231-240, 2017.

[79] K. P. Purnhagen and J. H. Wesseler, "Maximum vs. minimum harmonization: what to expect from the institutional and legal battles in the EU on gene editing technologies," Pest Management Science, vol. 75, no. 9, pp. 2310-2315, 2019.

[80] W. Parrott, "Outlaws, old laws and no laws: the prospects of gene editing for agriculture in United States," Physiologia Plantarum, vol. 164, no. 4, pp. 406-411, 2018.

[81] S. J. Smyth, "Regulation of genome editing in plant biotechnology: Canada," in Regulation of Genome Editing in Plant Biotechnology: A Comparative Analysis of Regulatory Frameworks of Selected Countries and the EU, pp. 111-135, Springer, Berlin, Germany, 2019.

[82] D. Kyetere, E. Okogbenin, J. Okeno et al., "The role and contribution of plant breeding and plant biotechnology to sustainable agriculture in Africa," Afrika Focus, vol. 32, pp. 83-108, 2019.

[83] J. Komen, L. Tripathi, B. Mkoko, D. O. Ofosu, H. Oloka, and D. Wangari, "Biosafety regulatory reviews and leeway to operate: case studies from sub-Sahara Africa," Frontiers in Plant Science, vol. 11, p. 130, 2020.

[84] S. Pillay and D. W. Thaldar, "CRISPR: challenges to south African biotechnology law," South African Journal of Bioethics and Law, vol. 11, no. 2, pp. 89-92, 2018.

[85] Z. Liang, K. Chen, Y. Zhang et al., "Genome editing of bread wheat using biolistic delivery of CRISPR/Cas9 in vitro transcripts or ribonucleoproteins," Nature Protocols, vol. 13, no. 3, pp. 413-430, 2018.

[86] D. D. Songstad, J. F. Petolino, D. F. Voytas, and N. A. Reichert, "Genome editing of plants," Critical Reviews in Plant Sciences, vol. 36, no. 1, pp. 1-23, 2017.

[87] F. Hartung and J. Schiemann, "Precise plant breeding using new genome editing techniques: opportunities, safety and regulation in the EU," The Plant Journal, vol. 78, no. 5, pp. 742-752, 2014.

[88] T. Ishii and M. Araki, "Consumer acceptance of food crops developed by genome editing," Plant Cell Reports, vol. 35, no. 7, pp. 1507-1518, 2016.

[89] K. Belhaj, A. Chaparro-Garcia, S. Kamoun et al., "Plant genome editing made easy: targeted mutagenesis in model and crop plants using the CRISPR/Cas system," Plant Methods, vol. 9, no. 39, 2013.

[90] A. Scheben and D. Edwards, "Bottlenecks for genome-edited crops on the road from lab to farm," Genome Biology, vol. 19, no. $178,2018$. 
[91] F. Wolter and H. Puchta, "Application of CRISPR/Cas to understand cis- and trans-regulatory elements in plants," in N. Yamaguchi, Plant Transcription Factors. Methods in Molecular Biology, vol. 1830, Methods in Molecular Biology, Humana Press, New York, NY, USA, 2018.

[92] S. Huang, D. Weigel, R. N. Beachy, and J. Li, "A proposed regulatory framework for genome-edited crops," Nature Genetics, vol. 48, no. 2, pp. 109-111, 2016. 